\title{
The effect of vitamin D3 supplementation on Interleukin-6 concentrations in healthy South Asian and Caucasian women: preliminary analysis of the D2-D3 study
}

\author{
A.L. Salter, O.A. Akinyemi, J. Elliott, J. Sier, J. Hunt, S.A. Lanham-New and D.J. Blackbourn \\ School of Biosciences and Medicine, Faculty of Health and Medical Sciences, University of Surrey, Guildford, Surrey, \\ $U K$, GU2 $7 X H$.
}

Low vitamin D levels have been associated with infectious disease such as tuberculosis ${ }^{(1)}$ and autoimmune disorders such as rheumatoid arthritis ${ }^{(2)}$. The expression of the vitamin D-receptor on cells of the innate and adaptive immune system implicates a role for vitamin D in immune function. Interleukin-6 (IL-6), an immune function marker, is an inflammatory cytokine whose production by human monocytes is inhibited by vitamin $\mathrm{D}$ in vitro ${ }^{(3)}$. The present study aimed to identify whether there are any changes in IL-6 concentrations during the winter months and the effect of vitamin D3 supplementation on IL-6 in South Asian and Caucasian women living in the UK.

A total of 70 subjects (South Asian $n=29$, Caucasian $n=41$ ) were selected from the D2-D3 study ${ }^{(4)}$ based on the greatest 25-hydroxyvitamin D (25OHD) changes across the 12 week winter intervention period. Plasma samples were taken at baseline, 6 and 12 weeks but only those from visit 1 (baseline) and visit 2 (6 weeks) were analysed in this study. IL-6 was quantified using highsensitivity enzyme-linked immunosorbent assay (R\&D Systems, Minneapolis, MN, USA) and all standards and samples were plated in duplicate. Serum 25OHD levels had previously been determined by HPLC-MS/MS and were therefore available for use in this study. The D2-D3 study had ethical approval from both the South-East Coast (Surrey) NHS Research Ethics Committee (NHS REC No. 11/LO/0708) and the University of Surrey Ethics Committee (Fast-Track EC/2011/97/FHMS).

In the placebo group, IL-6 concentration did not change significantly from visit 1 to visit 2 for either the South Asian or Caucasian women. Supplementation with vitamin D3 did not significantly affect IL-6 concentration from visit 1 to visit 2 in either the South Asian or Caucasian women (Table 1). 25OHD levels decreased significantly in the South Asian and Caucasian women taking the placebo and increased significantly in the women taking vitamin D3 (Table 2).

Table 1. Interleukin-6 (IL-6) concentrations at visit 1 (V1) and visit 2 (V2).

\begin{tabular}{|c|c|c|c|c|c|c|c|c|}
\hline & \multicolumn{4}{|c|}{ Placebo } & \multicolumn{4}{|c|}{ Vitamin D3 } \\
\hline & \multicolumn{2}{|c|}{ SA $(n=11)$} & \multicolumn{2}{|c|}{$\mathrm{CA}(\mathrm{n}=23)$} & \multicolumn{2}{|c|}{ SA $(n=18)$} & \multicolumn{2}{|c|}{$\mathrm{CA}(\mathrm{n}=18)$} \\
\hline & Median & IQR & Median & IQR & Median & IQR & Median & IQR \\
\hline IL-6 V1 (pg/mL) & 1.54 & 0.62 & 1.23 & 0.73 & 1.85 & 1.29 & 0.97 & 0.49 \\
\hline IL-6 V2 (pg/mL) & 1.63 & 0.93 & 1.29 & 0.83 & $2 \cdot 10$ & 1.54 & $1 \cdot 26$ & 1.68 \\
\hline$P$ value & $0 \cdot 859$ & & $0 \cdot 088$ & & $0 \cdot 071$ & & $0 \cdot 191$ & \\
\hline
\end{tabular}

$\mathrm{IQR}=$ Interquartile range, $\mathrm{SA}=$ South Asian, $\mathrm{CA}=$ Caucasian.

Table 2. 25-hydroxyvitamin D (25OHD) concentrations at visit 1 (V1) and visit 2 (V2).

\begin{tabular}{|c|c|c|c|c|c|c|c|c|}
\hline & \multicolumn{4}{|c|}{ Placebo } & \multicolumn{4}{|c|}{ Vitamin D3 } \\
\hline & \multicolumn{2}{|c|}{ SA $(n=12)$} & \multicolumn{2}{|c|}{$\mathrm{CA}(\mathrm{n}=23)$} & \multicolumn{2}{|c|}{ SA $(n=18)$} & \multicolumn{2}{|c|}{$\mathrm{CA}(\mathrm{n}=18)$} \\
\hline & Median & IQR & Median & IQR & Median & $\overline{\mathrm{IQR}}$ & Median & IQR \\
\hline $25 \mathrm{OHD} \mathrm{V1}(\mathrm{nmol} / \mathrm{L})$ & $17 \cdot 20$ & $19 \cdot 35$ & $58 \cdot 10$ & $47 \cdot 10$ & $15 \cdot 50$ & $20 \cdot 55$ & $33 \cdot 85$ & $15 \cdot 50$ \\
\hline 25OHD V2 (nmol/L) & $15 \cdot 30$ & $12 \cdot 05$ & $41 \cdot 50$ & $29 \cdot 20$ & $56 \cdot 55$ & $19 \cdot 35$ & $78 \cdot 30$ & $30 \cdot 32$ \\
\hline$P$ value & $0 \cdot 012^{*}$ & & $<0 \cdot 001^{*}$ & & $<0 \cdot 001^{*}$ & & $<0.001 *$ & \\
\hline
\end{tabular}

$* \mathrm{P}<0.05$ Wilcoxon Signed Rank Test, IQR = Interquartile range, SA = South Asian, CA = Caucasian.

The present study shows no significant change in IL-6 levels across the winter and no effect of vitamin D3 supplementation on IL-6 levels despite the significant change in vitamin D status of women in the D2-D3 study ${ }^{(4)}$. The present study supports a meta-analysis which found that vitamin D supplementation had no significant effect on IL- 6 concentrations ${ }^{(5)}$ as well as a 12 week study that showed no effect of calcium/vitamin D3 treatment on IL-6 concentrations ${ }^{(6)}$. Although these findings do not support a beneficial role for vitamin D with respect to IL-6 concentrations, new data in our labs with regard to IL-8 may warrant further study ${ }^{(7)}$.

1. Huang S, Wang X, Liu Z et al. (2017) Drug Des Devel Ther 11, 91-102.

2. Kostoglou-Athanassiou I, Athanassiu P, Lyraki A et al. (2012) Ther Adv Endocrinol Metab 3(6), 181-187.

3. Zhang Y, Leung DYM, Richers BN et al. (2012) J Immunol 188(5), 2127-2135.

4. Tripkovic L, Wilson LR, Hart K et al. (2017) Am J Clin Nutr 106(2), 481-490.

5. Jamka M, Woźniewicz M, Walkowiak J et al. (2016) Eur J Nutr 55(6), 2163-2176.

6. Gannagé-Yared MH, Azoury M, Mansour I et al. (2003) J Endocrinol Invest 26(8), 748-753.

7. Akinyemi OA et al. (2017). 